\title{
Role of the Transcriptional Activator XInR of Fusarium oxysporum in Regulation of Xylanase Genes and Virulence
}

\author{
Fernando Calero-Nieto, Antonio Di Pietro, M. Isabel G. Roncero, and Concepcion Hera \\ Departamento de Genetica, Universidad de Córdoba, Campus de Rabanales, C-5, 14071 Córdoba, Spain
}

Submitted 23 November 2006. Accepted 11 March 2007.

Fungal infection of plants involves degradation of the host cell wall through the action of lytic enzymes secreted by the pathogen. The role of these enzymes in virulence is difficult to determine due to their functional redundancy and, therefore, remains controversial. Here, we have studied XInR, a zinc-finger transcription factor from the vascular wilt pathogen Fusarium oxysporum that is orthologous to the major transcriptional activator of xylanase genes in Aspergillus spp. Transcription of the $x \ln R$ gene was activated by inducing carbon sources such as oat spelt xylan (OSX) and repressed by glucose. Targeted knockout of $x \ln R$ in $F$. oxysporum resulted in lack of transcriptional activation of structural xylanase genes, both in culture and during infection of tomato plants, as well as in dramatically reduced extracellular xylanase activity. By contrast, overexpression of $x \ln R$ under the control of the Aspergillus nidulans gpdA promoter did not significantly increase xylanase activity, suggesting that $X \ln R$ is regulated not only at the transcriptional but also at the post-translational level. The $\Delta x \ln R$ mutants were still fully virulent on tomato plants. Thus, XInR, the major transcriptional activator of xylanase genes, is not an essential virulence determinant in F. oxysporum.

Plant cell walls consist mainly of polysaccharides such as cellulose, hemicellulose, and pectin. Degradation of plant cell wall polymers by saprophytic and pathogenic fungi is a complex process involving the synergistic action of different classes of extracellular enzymes. It has been suggested that plant cell-wall-degrading enzymes not only provide fungal pathogens with a means of obtaining nutrients but also contribute actively to the infection process by breaking down the structural barriers, thus facilitating fungal invasion (Walton 1994). One of the major polysaccharide components of secondary plant cell walls, particularly in the graminaceae, is xylan. This polymer consists of a $\beta$-1,4-linked D-xylose backbone that can be substituted by different side groups such as L-arabinose, D-galactose, acetyl, feruloyl, p-coumaroyl, and glucuronic acid residues. The structure of xylan is highly complex and its complete hydrolysis requires the coordinated action of

\section{Corresponding author: Concepcion Hera; E-mail: ge1hedic@uco.es}

The GenBank accession number of the Fusarium oxysporum $x \ln R$ gene is EF057395.

* The $\boldsymbol{e}$-Xtra logo stands for "electronic extra" and indicates that supplemental material is available online. An additional table and a figure are published online. an array of different enzymes (de Vries and Visser 2001). Among these, the endo- $\beta-1,4-x y l a n a s e s$ play a key role in $x y-$ lan depolymerization due to their ability to break down the backbone of the xylan molecule (Wong et al. 1988). The role of endo- $\beta-1,4$-xylanase genes in fungal virulence has been examined by targeted knockout in two cereal pathogens, Cochliobolus carbonum and Magnaporthe grisea. The outcome of these studies indicates that the deletion of individual endo- $\beta$ 1,4-xylanases has no detectable effect on the virulence of these pathogens (Apel et al. 1993; Apel-Birkhold and Walton 1996; Wu et al. 2006). By contrast, deletion of an endoxylanase gene in Botrytis cinerea significantly reduced virulence on grape plants and tomato leaves, delaying the appearance of secondary lesions and reducing the lesion size by more than $70 \%$ (Brito et al. 2006). In addition to their role in virulence, fungal endoxylanases also can act as elicitors of the plant defense system (Belien et al. 2006).

Our group is studying the role of cell-wall-degrading enzymes in the vascular wilt pathogen Fusarium oxysporum. This fungus attacks over a hundred different plant species and serves as a useful model for studying pathogenicity mechanisms in soilborne root pathogens (Di Pietro et al. 2003). Four genes ( $x y l 2$, $x y l 3, x y l 4$, and $x y l 5)$ encoding putative endo- $\beta-1,4$-xylanases of the tomato pathogen $F$. oxysporum $\mathrm{f}$. sp. lycopersici have been cloned, and three of these ( $x y l 3,-4$, and -5 ) have been deleted by targeted gene knockout. Similarly as in the monocot pathogens $C$. carbonum and $M$. grisea, inactivation of individual xylanase genes in $F$. oxysporum did not affect virulence on tomato plants (Gómez-Gómez et al. 2001, 2002). Thus, either endo- $\beta-1,4-$ xylanases do not play an important role during infection in these fungal pathogens or structurally similar genes that are present in the fungal genomes can functionally compensate for the loss of single structural xylanase genes (Walton 1994).

One way to overcome the problem of functional redundancy is to abolish the expression of an entire class of cell-walldegrading enzymes by knocking out components of signal transduction pathways (Tonukari et al. 2000) or transcriptional activators. The transcription factor XlnR originally was identified in Aspergillus niger as a transcriptional activator of $\mathrm{xy}$ lanolytic genes (van Peij et al. 1998b), and its orthologues were shown to perform similar functions in other saprophytic species such as A. oryzae and Trichoderma reesei (Marui et al. 2002; Rauscher et al. 2006). The XlnR orthologues control transcription of not only xylanolytic genes but also endoglucanase and cellobiohydrolase genes, suggesting a general role in cellulose and hemicellulose degradation and metabolism (de Vries and Visser 1999; Gielkens et al. 1999; Rauscher et al., 2006; Stricker et al., 2006; van Peij et al. 1998a). In agreement with such a role, expression of the $x y r R$ gene encoding D-xylose 

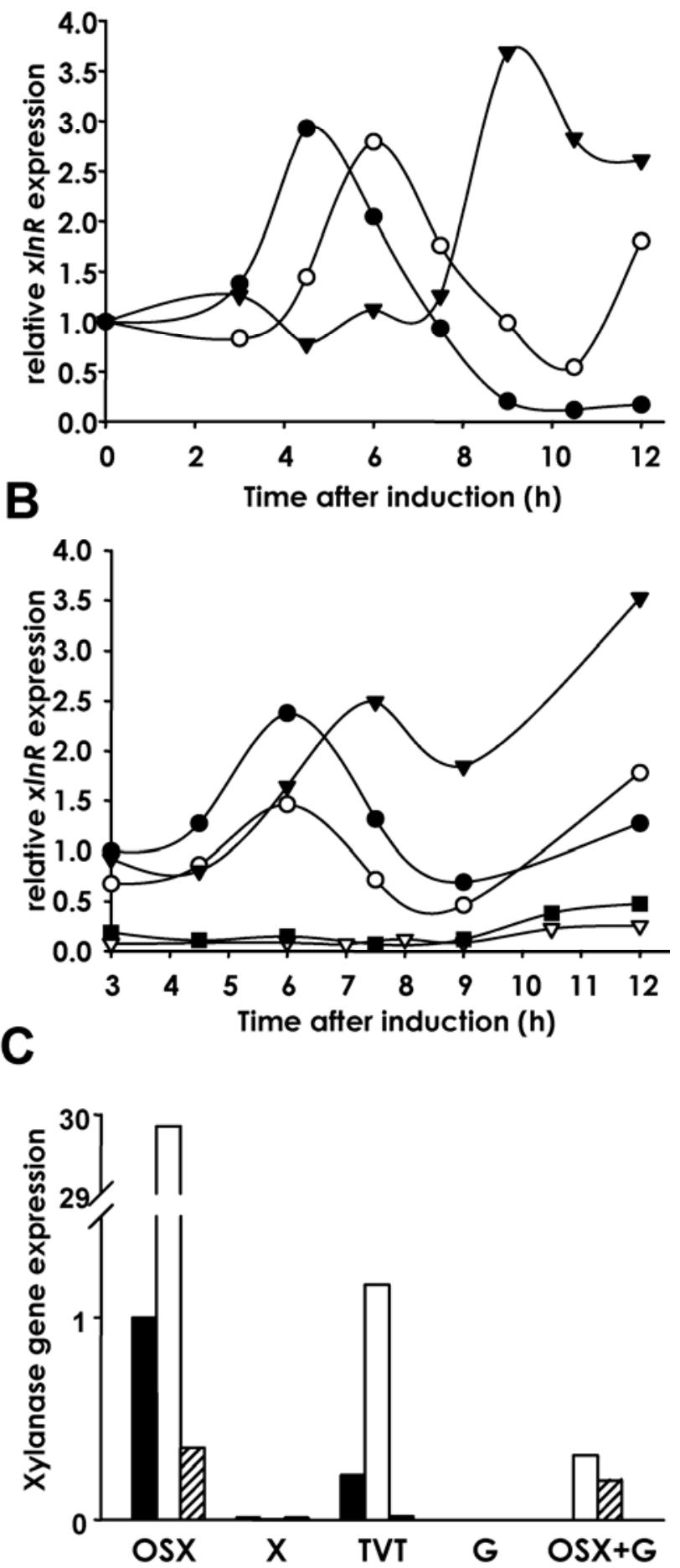

Fig. 1. Real-time polymerase chain reaction (PCR) quantification of transcript accumulation in mycelia grown on different carbon sources or at different ambient $\mathrm{pH}$ values. A, Accumulation of $x \ln R$ transcripts in mycelia grown on synthetic medium (SM) supplemented with $1 \%$ oat spelt xylan (OSX) and buffered at pH 5.5 (solid circles), 6.5 (empty circles), or 8.0 (inverted triangles). B, Accumulation of $x \ln R$ transcripts in mycelia grown on SM buffered at $\mathrm{pH} 6.5$ and supplemented with the following carbon sources: $1 \%(\mathrm{wt} / \mathrm{vol})$ OSX (solid circles), $0.1 \%$ xylose (X, solid inverted triangles), $1 \%$ tomato vascular tissue (TVT, empty circles), $1 \%$ glucose (G, solid squares), or $1 \%$ OSX $+1 \%$ glucose (OSX+G, empty inverted triangles). C, Accumulation of xylanase gene transcripts in mycelia grown for $24 \mathrm{~h}$ on SM supplemented with the indicated carbon sources. Transcripts measured were $x y l 3$ (black bars), xyl4 (empty bars), and $x y l 5$ (striped bars). Transcript levels are expressed relative to those of $x y l 3$ on OSX (set to 1). All values were normalized against those of the actin gene. reductase, an intracellular enzyme involved in D-xylose catabolism, also is regulated by $\mathrm{X} \operatorname{lnR}$ (Hasper et al. 2000).

In the present work, we cloned the $x \ln R$ orthologue of $F$. $o x$ ysporum $\mathrm{f}$. sp. lycopersici and studied its function by generating $\Delta x \ln R$ loss-of-function mutants as well as strains carrying an $x \ln R$ allele under the control of a strong constitutive promoter. Comparative analysis of the wild-type and mutant strains by real-time quantitative reverse-transcriptase polymerase chain reaction (RT-PCR) showed that XlnR is a transcriptional activator of xylanase genes in F. oxysporum. Virulence of the mutants on tomato plants was indistinguishable from that of the wild-type strain, indicating that XlnR is dispensable for pathogenicity in $F$. oxysporum.

\section{RESULTS}

Characterization of the $x \ln R$ gene of $F$. oxysporum.

The $F$. oxysporum $x \ln R$ orthologue was cloned and sequenced (details below). Analysis of the genomic sequence revealed the existence of two in-frame ATG codons that could act as a possible translation start points. The first ATG was considered as the most likely translation start point in the $F$. graminearum $x \ln R$ orthologue (Fusarium graminearum Sequencing Project, Broad Institute), according to the automated annotation with the FGENESH program (Softberry Inc.). Two independent $x \ln R$ clones were isolated from a cDNA library and sequenced, allowing the identification of the putative transcription start site at position -856 relative to the first ATG. Comparison of the genomic and cDNA sequences revealed the presence of three introns, two within the open reading frame (ORF) (46 and $52 \mathrm{bp}$ in length) and one in the $5^{\prime}$ untranslated region (404 $\mathrm{bp})$, at position -550 to -146 relative to the putative start codon. Putative consensus TATA sequences $(-889,-441$, and 378) and multiple CAAAT boxes were identified. Four CCAAT sequences, the putative binding sites for the Hap complex in filamentous fungi (Brakhage et al. 1999), were present at positions $-1,671,-938,-769$, and -304 . Putative stress response elements (CCCCT) were detected at positions -235 and -118 , as well as putative binding sequences of the carbon catabolite repressor CreA. Interestingly, two sequences (GGCTAA) reported as consensus binding sites for the XlnR protein (Marui et al., 2002; van Peij et al. 1998b) were located at positions -992 and -600 . Southern analysis of total genomic DNA treated with different restriction enzymes suggested that $x \ln R$ is a single-copy gene (data not shown). In agreement with this result, a BLASTP search of the complete genome database of the closely related species $F$. graminearum detected one significant alignment showing a very high degree of identity with F. oxysporum (BLASTP value 0.0 ).

The deduced XlnR protein contained 938 amino acids and had a predicted molecular mass of $103 \mathrm{kDa}$ and a isoelectric point of 6.1. Analysis of the amino acid sequence revealed significant homology to XlnR proteins of A. niger, A. nidulans, and A. oryzae (Marui et al. 2002 ; van Peij et al. 1998b) (Supplemental Figure 1). A binuclear zinc cluster with similarity to the DNA binding domain of the Saccharomyces cerevisiae GAL4 protein (Marmorstein and Harrison 1994) was identified at the N-terminal region (96 to 122). An Arg-Arg-Arg motif reminiscent of that involved in DNA binding of the $A$. nidulans AlcR transcriptional regulator (Nikolaev et al. 1999) was located in the $\mathrm{N}$-terminal region adjacent to the binuclear zinc cluster and two putative coiled-coil regions were present immediately downstream of the binuclear zinc cluster and in the C-terminal region, respectively. The sequence RRRIWW resembling the motif RRRLWW related to inhibition (Suárez et al. 1995) was found within a region similar to the so-called middle homology region (Schjerling and Holmberg 1996). 
Transcriptional regulation of xylanolytic genes in F. oxysporum.

Thus far, four different xylanase genes have been identified in $F$. oxysporum whose expression is regulated by carbon source and ambient pH (Gómez-Gómez et al. 2001, 2002; Ruiz-Roldán et al. 1999). To obtain a detailed picture of the regulation of the $F$. oxysporum xylanolytic system, transcript accumulation of the $x \ln R$ gene and of three structural endo- $\beta$ 1,4-xylanase genes ( $x y l 3, x y l 4$, and $x y l 5)$ was quantified in mycelia grown on different carbon sources and ambient $\mathrm{pH}$ values, using real-time RT-PCR. Transcript levels of the $x \ln R$ gene increased three- to fourfold after transfer to oat spelt $x y$ lan (OSX) (Fig. 1A). This first increase was transient, and was followed by a marked decrease in expression and by a second increase of transcript levels at later stages of growth (Fig. 1A and B). No significant quantitative differences were detected between mycelia grown at different ambient $\mathrm{pH}$ values, although the lag phase before induction increased at higher $\mathrm{pH}$. A similar retardation effect at alkaline $\mathrm{pH}$ also was observed in the expression of the structural xylanase genes $x y l 3, x y l 4$, and $x y l 5$ (data not shown). In subsequent experiments, $\mathrm{pH} 6.5$ was used for being similar to that of tomato plant tissue.

Among the carbon sources tested, OSX, xylose, and tomato vascular tissue, but not glucose, induced expression of $x \ln R$ (Fig. 1B). Moreover, induction of $x \ln R$ by OSX was completely abolished in the presence of $1 \%$ glucose, suggesting a mechanism of catabolite repression. A similar carbon source effect was detected on expression of $x y l 3, x y l 4$, and $x y l 5$, except that $0.1 \%$ xylose did not induce expression of these structural xylanase genes (Fig. 1C). Among the three xylanase genes studied, xyl4 showed the highest levels of induction.

The course of events leading to induction of xylanolytic activity after transfer to OSX is shown in Figure 2. The transient upregulation of $x \ln R$ after $6 \mathrm{~h}$ is followed with a delay of $2 \mathrm{~h}$ by transcriptional activation of $x y l 3, x y l 4$, and $x y l 5$ (Fig. 2A). Concomitant with the induction of the structural xylanase genes, a rapid increase in specific xylanase activity can be observed in the supernatant (Fig. 2B). This xylanase activity results first in the release of intermediate products of hydrolysis from the xylan backbone and, subsequently, in the accumulation of considerable amounts of xylose, starting at 9 $\mathrm{h}$ after induction (Fig. 2C). Collectively, these results suggest that the transcriptional regulator $x \ln R$ and the xylanase genes $x y l 3, x y l 4$, and $x y l 5$ of $F$. oxysporum are subject to substrate induction and carbon catabolite repression, and that $x \ln R$ induction precedes that of the structural xylanase genes.

\section{$\mathrm{X} \ln R$ is required for xylanolytic activity and xylanase gene expression in $F$. oxysporum.}

To examine the role of XlnR in F. oxysporum, two types of alleles, a loss-of-function allele and a constitutively expressed allele, were generated (Fig. 3). Mutants carrying a disrupted copy of the $x \ln R$ gene were obtained by gene replacement of the wild-type allele with the $\Delta x \ln R$ allele (Fig. 3A) (details below). Southern blot analysis of 45 transformants identified 1 hygromycin-resistant transformant in which the 3.3-kb BamHI fragment on the wild-type strain had been lost (Fig. 3B). RTPCR analysis confirmed the absence of the $x \ln R$ transcript in this mutant, which was named $\Delta x \ln R$ (Fig. 3C).

To confirm that the phenotype of the $\Delta x \ln R$ mutant was indeed due to loss of XlnR function, a 5.2-kb fragment containing the complete $x \ln R$ gene was introduced into the $\Delta x \ln R$ mutant by cotransformation with the phleomycin resistance marker. Phleomycin-resistant transformants were analyzed for presence of a functional $x \ln R$ allele by Southern analysis. Several transformants contained the expected 3.3-kb BamHI fragment present in the wild-type strain and one of these was selected for further experiments and named $\Delta x \ln R+x \ln R$ (Fig. 3B).

A constitutively expressed $x \ln R$ allele $(P g p d a:: x \ln R)$ was generated by fusing the strong constitutive $A$. nidulans gpdA promoter to the $x \ln R$ ORF (Fig. 3A) (details below). The construct was introduced into the wild-type strain and hygromycin-resis-

A

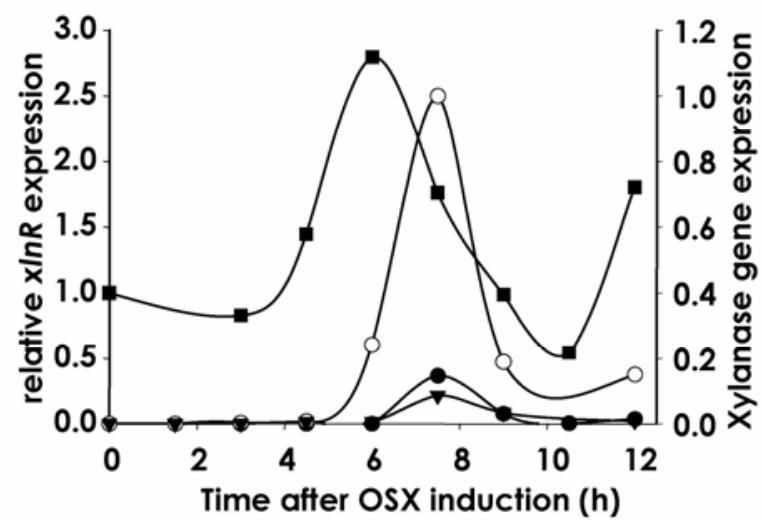

B

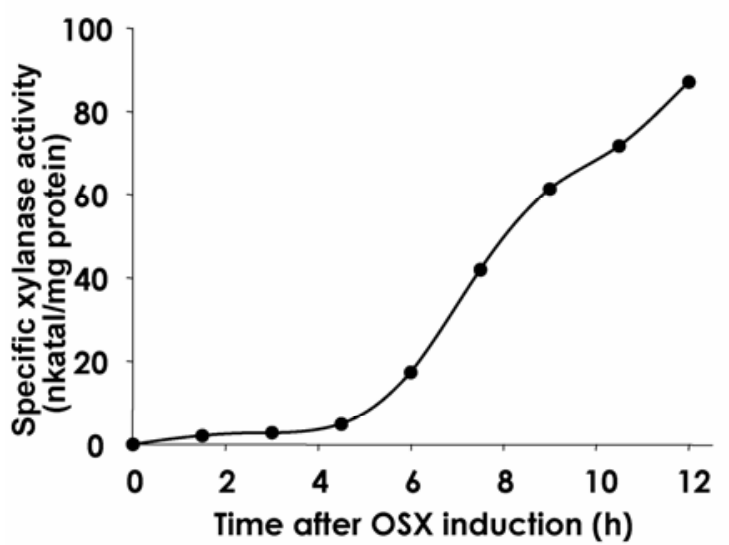

C

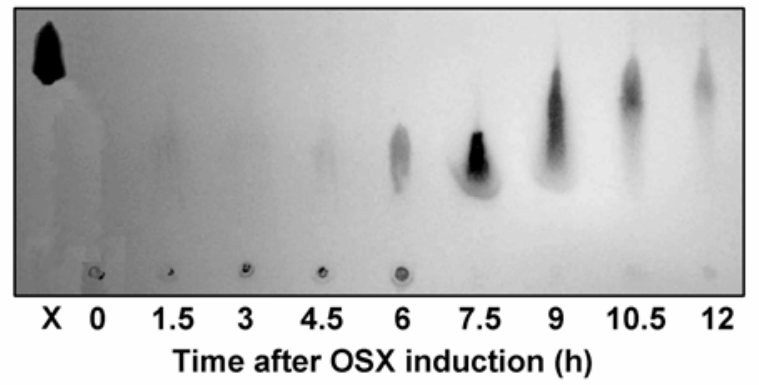

Fig. 2. Time course of the activation of the xylanolytic system in Fusarium oxysporum upon induction by $1 \%$ oat spelt xylan (OSX). A, Real-time reverse-transcriptase polymerase chain reaction quantification of transcript accumulation of $x \ln R$ (solid squares), $x y l 3$ (solid circles), $x y l 4$ (empty circles), and $x y l 5$ (solid triangles). Levels of $x \ln R$ transcripts are expressed relative to time $0 \mathrm{~h}$. Xylanase gene transcripts are expressed relative to xyl4 at time $7.5 \mathrm{~h}$. B, Specific xylanase activity in OSX-grown culture supernatants at different times after induction. C, Analysis of hydrolysis products. Aliquots of OSX-grown culture supernatants were removed at the indicated times, subjected to thin-layer chromatography, and sprayed with aniline phenylamine as detection reagent. D-xylose (X) was used as standard. 
tant transformants were analyzed by Southern blot. Multiple transformants contained additional hybridizing BamHI fragments. One of these transformants, which exhibited two additional hybridizing bands, indicative of a single ectopic insertion of the construct (Fig. 3B), was selected for further experiments and named $x \ln R^{C}$. Real-time RT-PCR detected dramatically higher accumulation of $x \ln R$ transcript levels in the $x \ln R^{C}$ strain compared with the wild type (Fig. 3D). As expected for the constitutive $\operatorname{PgpdA}$ promoter, the $x \ln R^{C}$ strain produced significant levels of $x \ln R$ transcript even on noninducing carbon sources such as glucose or OSX + glucose, although the highest levels were detected in OSX, probably due to the contribution from the inducible wild-type $x \ln R$ allele (Fig. 3D). Thus, $x \ln R$ expression in the $x \ln R^{C}$ strain is strongly upregulated but still inducible by OSX.

When grown on plates containing AZO-xylan, both the wildtype strain and the complemented mutant produced a clear halo around the colony, whereas the $\Delta x \ln R$ mutant did not (Fig. 4A). The $x \ln R^{C}$ strain produced a halo of similar size to that of the wild type (results not shown). In the culture supernatant of the $\triangle x \ln R$ mutant grown on OSX, the undegraded xylan was clearly visible as a precipitate, but this was not the case in the wild-type strain (Fig. 4B). In agreement with this result, specific xylanase activity in culture supernatants of the $\Delta x \ln R$ mutant grown on OSX or tomato vascular tissue was reduced dramatically compared with the wild type, and that of the $x \ln R^{C}$ strain was comparable with the wild type (Fig. 4D). Likewise, the strong and transient increase in $x y l 3$ transcript levels after transfer to OSX was observed only in the wild type and the $x \ln R^{C}$ strain, but not in the $\Delta x \ln R$ mutant (Fig. 4E).

The effect of mutations in $x \ln R$ on cellulase activity of $F$. oxysporum was tested by determining production of a clear halo on plates containing carboxymethyl cellulose. The wild-type strain, the $\Delta x \ln R$ mutant, and the $x \ln R^{C}$ strain produced halos of similar size, indicating that $\mathrm{X} \ln \mathrm{R}$ is not required for the production of carboxymethyl cellulose-degrading activity under these conditions (Fig. 4C).

\section{XInR controls xylanase gene expression}

during infection but is not essential for virulence.

To study induction of the xylanolytic system during plant infection by $F$. oxysporum, transcript levels of $x \ln R, x y l 3, x y l 4$,
A

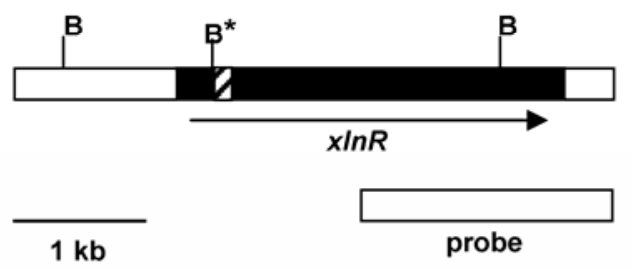

$\mathrm{pDx} \ln R$
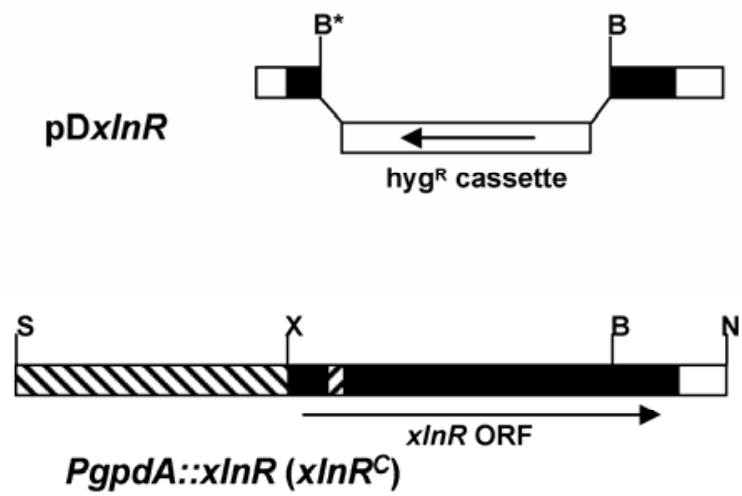

C

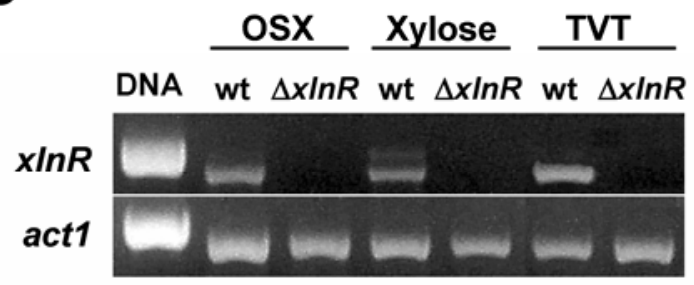

B

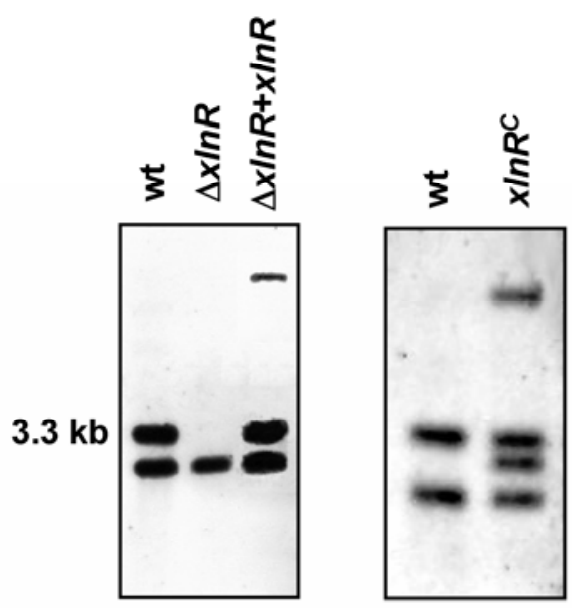

D

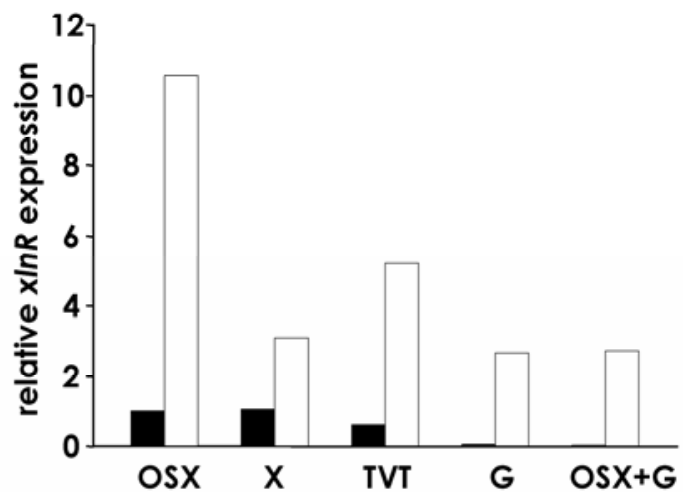

Fig. 3. Targeted disruption and constitutive expression of the $x \ln R$ gene in Fusarium oxysporum. A, Physical maps of the $x \ln R$ locus, the gene replacement vector pDxlnR, and the construct PgpdA::xlnR. B = BamHI, N = NotI, S = SalI, and X = XbaI. Direction of transcription is indicated by an arrow. The BamHI site introduced by site-directed mutagenesis is marked with an asterisk. The probe used for Southern analysis is indicated. B, Southern blot analysis of different $F$. oxysporum strains. Genomic DNA from the indicated strains was treated with BamHI and the blot was hybridized with the labeled $x \ln R$ probe indicated in A. wt = wild type. The size of the BamHI fragment replaced in the mutant is shown on the left. C, Reverse-transcriptase polymerase chain reaction (RT-PCR) analysis for $x \ln R$ transcript accumulation in the wild-type (wt) and the $\Delta x \ln R$ mutant grown on the indicated carbon sources OSX $=$ oat spelt xylan; TVT = tomato vascular tissue). The actin gene was used as positive control. D, Real-time RT-PCR quantification of $x \ln R$ transcript accumulation in mycelia of the wild-type (black bars) and the $x \ln R^{C}$ strain (empty bars) grown for $6 \mathrm{~h}$ on synthetic medium with the indicated carbon sources. Transcript levels are expressed relative to wild-type strain on OSX. 
A
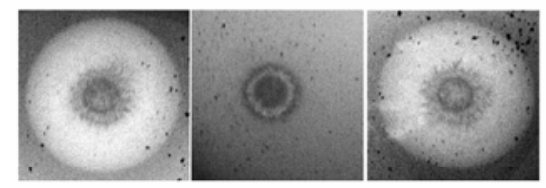

B

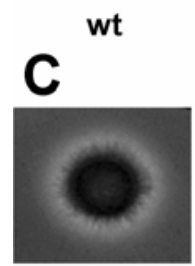

$\Delta x \ln R \quad \Delta x \ln R+x \ln R$

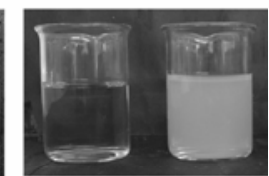

wt $\quad \Delta x \ln R$

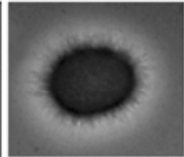

$\Delta x \ln R$

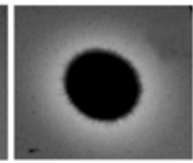

$x \ln R^{C}$

D

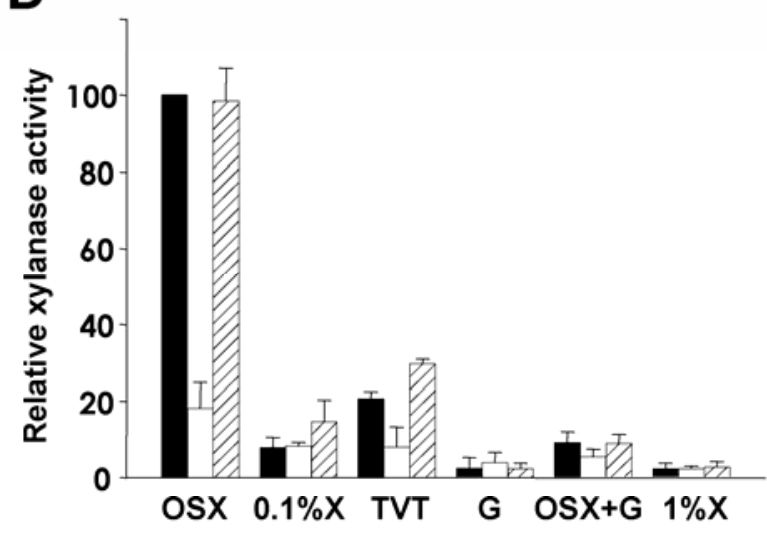

$\mathbf{E}$

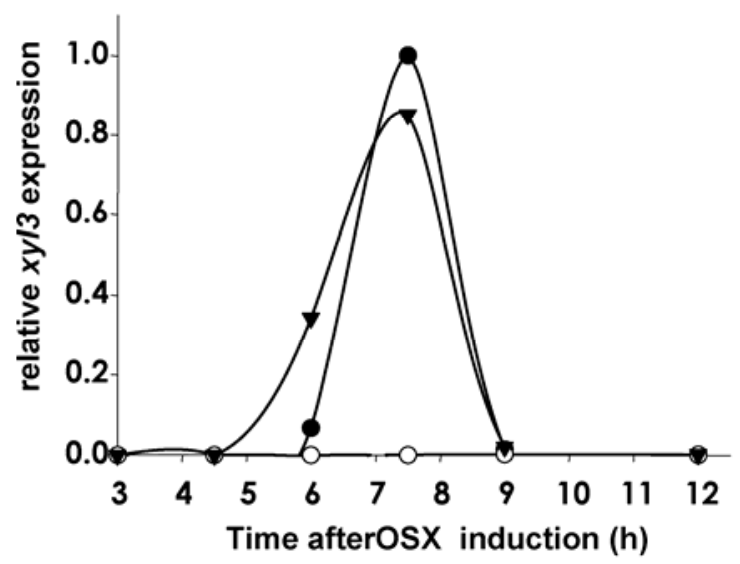

Fig. 4. XlnR regulates xylanolytic activity and xylanase gene expression in Fusarium oxysporum. A, Clear halo production on $0.15 \%$ azoxylan-containing plates for visualization of extracellular xylanase activity. The indicated strains were point inoculated and allowed to grow for 3 days at $28^{\circ} \mathrm{C}$; wt $=$ wild type. B, Aspect of supernatants from cultures of the indicated strains grown for $24 \mathrm{~h}$ on synthetic medium (SM) supplemented with $1 \%$ oat spelt xylan (OSX). The presence of undegraded xylan in the supernatant of the $\Delta x \ln R$ mutant is visible as a white precipitate. $\mathbf{C}$, Clear halo production on $0.5 \%$ carboxymethyl cellulose-containing plates for visualization of extracellular cellulase activity. The indicated strains were point inoculated and allowed to grow for 3 days at $28^{\circ} \mathrm{C}$, and the plates were stained with $0.1 \%$ Congo Red. D, Specific xylanase activities in culture supernatants of the wild-type (black bars), $\Delta x \ln R$ (empty bars), and $x \ln R^{C}$ strain (striped bars) grown for $24 \mathrm{~h}$ on SM supplemented with the indicated carbon sources. Activity is expressed as percentage of that of the wild-type strain grown on OSX. Error bars represent standard deviations calculated from three independent experiments. E, Real-time reverse-transcriptase polymerase chain reaction analysis of the time course of $x y l 3$ transcript accumulation in mycelia of the same strains as in $\mathbf{D}$ grown on SM with 1\% OSX. Transcript levels are expressed relative to the wild-type strain at $7.5 \mathrm{~h}$.

and $x y l 5$ were quantified by real-time RT-PCR, using total RNA extracted from roots and stems of tomato plants at different times after inoculation with the wild-type strain, the $\Delta x \ln R$ mutant, or the $x \ln R^{C}$ strain (Fig. 5). RNA from uninoculated plants was used as a control. Transcript levels of $x \ln R$ were low during the entire course of the experiment and only showed a significant increase in roots during the final stages of disease (Fig. 5A). Expression of the structural xylanase genes $x y l 3$ and $x y l 4$ followed a similar pattern (Fig. 5B and C),
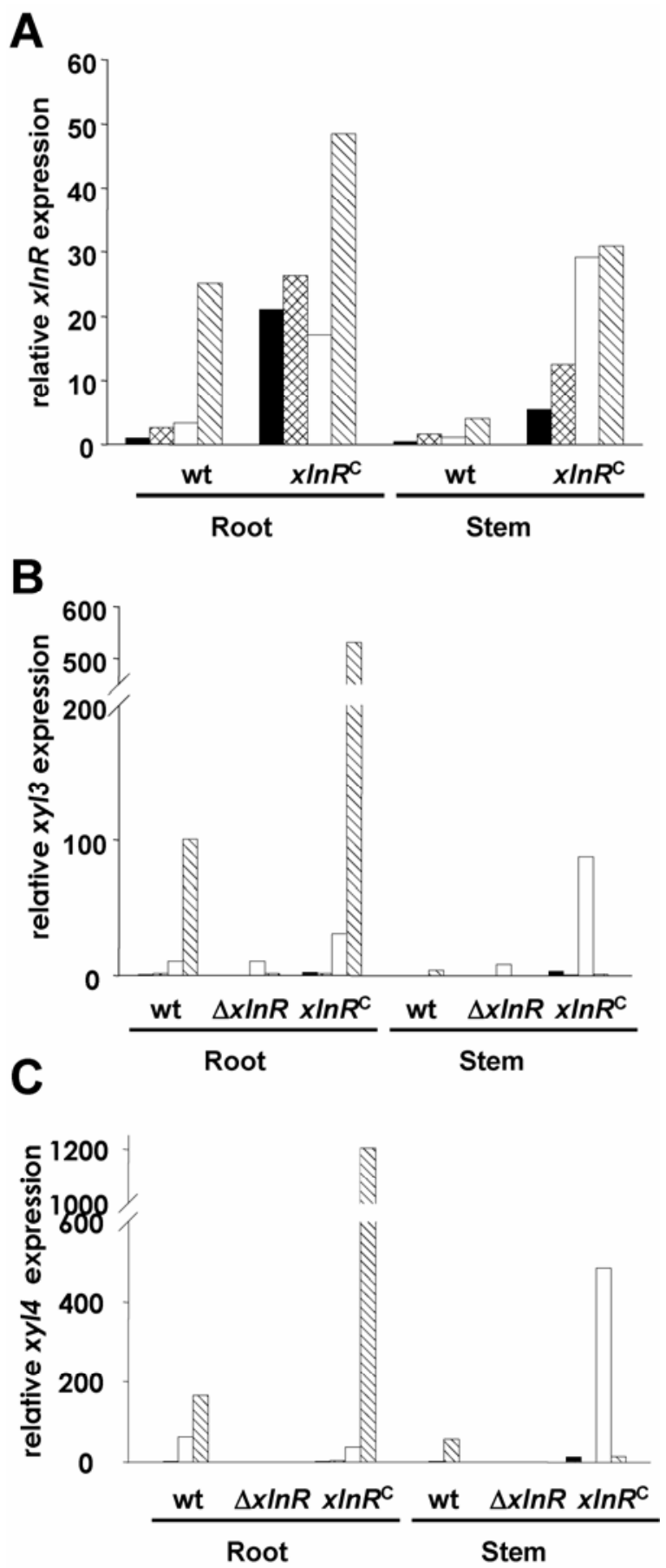

Fig. 5. XlnR controls expression of xylanase genes during infection of tomato plants. Real-time polymerase chain reaction quantification of transcript accumulation of $\mathbf{A}, x \ln R, \mathbf{B}, x y l 3$, and $\mathbf{C}, x y l 4$ was carried out with total RNA isolated from roots or lower stems of infected tomato plants at the following times after inoculation: 4 days (black bars), 7 days (hatched bars), 10 days (empty bars), and 14 days (striped bar). Transcript levels are expressed relative to the wild-type strain at 4 days after inoculation. 
whereas $x y l 5$ transcript levels were very low throughout the entire infection cycle (results not shown). $X \ln R$ transcript levels were significantly higher in the $x \ln R^{C}$ strain during all stages of infection, and this was paralleled by a strong increase in expression of $x y l 3$ and $x y l 4$, especially during the final stages of infection (Fig. 5B and C). By contrast, expression of $x y l 3$ and $x y l 4$ was basically undetectable in the $\Delta x \ln R$ mutant. These results indicate that expression of $x y l 3$ and $x y l 4$ genes is controlled by XlnR during infection.

The capacity of the strains to grow invasively on tomato fruit tissue was assayed by injecting a microconidial suspension directly into the fruit. The wild-type strain efficiently colonized and macerated the fruit tissue, producing aerial mycelium on the surface (Fig. 6A). Tissue maceration and mycelial growth in the $\Delta x \ln R$ mutant and the $x \ln R^{C}$ strain were similar to that of the wild type. To test the role of $X \ln R$ in virulence of $F$. oxysporum, infection assays were performed by immersing roots of tomato plants in microconidial suspensions. Severity of wilt symptoms in plants inoculated with the wild-type strain increased steadily throughout the experiment (Fig. 6B). Initially, leaf tips turned yellow and curved, then the stalk weakened and decayed, and the plants were dead 20 days after inoculation. Progression of disease symptoms in plants inoculated with the $\Delta x \ln R$ mutant or the $x \ln R^{C}$ strain was not significantly different from that of the wild-type strain, suggesting that $\mathrm{X} \operatorname{lnR}$ is not essential for virulence of $F$. oxysporum.

\section{DISCUSSION}

\section{$\mathrm{X} \ln \mathrm{R}$ is the major transcriptional activator} of xylanase genes in $F$. oxysporum.

In the present study, we have examined the role of the $x \ln R$ gene in the soilborne plant pathogen $F$. oxysporum. The deduced $x \ln R$ gene product has approximately $50 \%$ of overall identity with XlnR from A. niger, the major transcriptional activator of xylanolytic and cellulolytic genes in this species (van Peij et al. 1998a), and contains a zinc binuclear cluster DNA-binding domain and two coiled-coil regions similar to those of the $\mathrm{As}$ pergillus XlnR. In agreement with these structural analogies, our results suggest that $F$. oxysporum XlnR also functions as a major transcriptional activator of xylanase genes during both saprophytic and infectious growth. This view is supported by several lines of evidence. First, the transitory increase of $x \ln R$ transcript upon transfer to the inducing carbon source OSX preceded that of the structural xylanase genes $x y l 3, x y l 4$, and $x y l 5$ by approximately $2 \mathrm{~h}$. Such an expression pattern would be expected for a transcriptional activator whose own transcription is positively regulated by the inducer. This mechanism ensures that high amounts of $\mathrm{X} \operatorname{lnR}$ protein are present under inducing conditions to trigger transcription of the target genes, resulting in amplification of the activation signal.

Second, the promoters of the $x y l 3, x y l 4$, and $x y l 5$ xylanase genes of $F$. oxysporum contain at least one copy of the $\mathrm{X} \ln \mathrm{R}$ consensus-binding sequence GGCTAR previously described in A. niger (van Peij et al. 1998a). Moreover, the $x \ln R$ promoter itself also contains a copy of this consensus binding sequence, suggesting that $F$. oxysporum $\mathrm{X} \ln \mathrm{R}$ controls gene expression by binding to the promoters of structural xylanase genes but also to its own promoter. Indeed, electrophoretic mobility shift assay (EMSA) analysis revealed specific binding of recombinant $F$. oxysporum XlnR protein to a GGCTAR element present in the $x y l 4$ promoter (F. Calero-Nieto, C. Hera, A. Di Pietro, M. Orejas, and M. I. G. Roncero, unpublished data). In Aspergillus spp., the XlnR consensus-binding sequence is found not only in the promoters of xylanase genes but also in those of many hemicellulase and cellulase genes (van Peij et al. 1998a). Whether this is also the case in F. oxysporum remains to be de- termined once the complete genome sequence of the species becomes available.

Third and most importantly, the $\Delta x \ln R$ loss-of-function mutant failed to activate xylanase gene expression both during saprophytic growth on OSX and during infection of tomato plants. The knockout mutant also showed dramatically reduced total extracellular xylanase activity, strongly suggesting that XlnR controls transcription of not only $x y l 3, x y l 4$, and $x y l 5$ but also that of other xylanase genes expressed by $F$. oxysporum under the conditions studied. Taken together, these results provide strong evidence that $\mathrm{X} \ln \mathrm{R}$ is the major transcriptional activator of xylanase genes in F. oxysporum.

In Aspergillus and Trichoderma spp., XlnR also regulates cellulase gene expression (Stricker et al. 2006; van Peij et al. 1998a). We did not detect significant differences in the capacity of the wild-type and $\Delta x \ln R$ strains to produce a clear halo on carboxymethylcellulose. Although this outcome suggests that $\mathrm{XlnR}$ is not essential for cellulase production by $F$. oxysporum under these conditions, it is possible that $\mathrm{X} \operatorname{lnR}$ may regulate cellulase gene expression under different environmental conditions.

\section{Mechanism of XInR activation.}

The exact mechanism through which XlnR becomes activated to promote xylanase gene expression is unknown. Our results

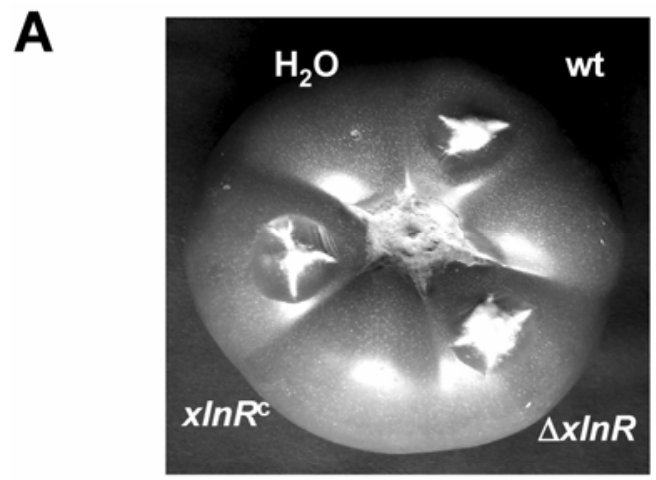

B

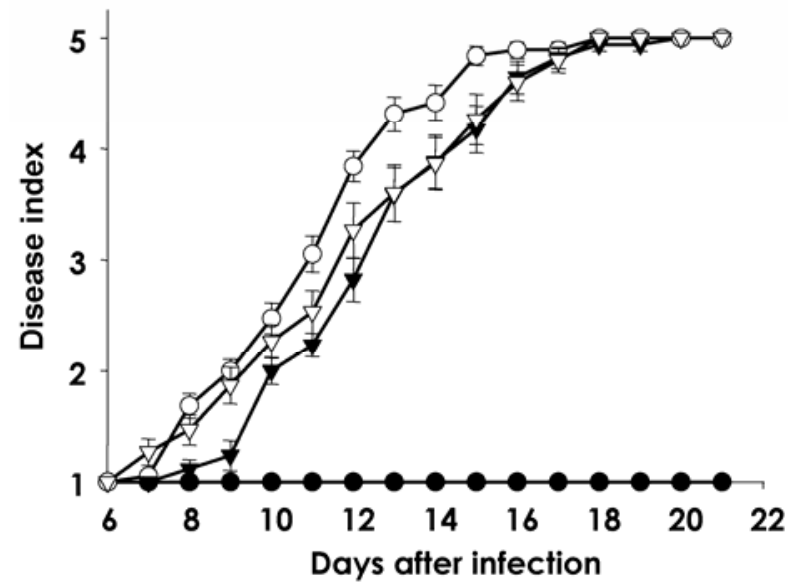

Fig. 6. $X \ln R$ is not required for virulence of Fusarium oxysporum. A, Invasive growth on tomato fruit. Inoculation was performed by injecting $5 \times$ $10^{5}$ microconidia of the indicated strains. The photograph was taken after 4 days of incubation at $28^{\circ} \mathrm{C}$ and $100 \%$ relative humidity; $w t=$ wild type. B, Incidence of Fusarium wilt after root inoculation of tomato plants (cv. Vemar). Severity of disease symptoms was recorded at the indicated times after inoculation using an index ranging from 1 (healthy plant) to 5 (dead plant). Symbols refer to plants inoculated with the following strains: wildtype (open circles), $\Delta x \ln R$ (solid inverted triangles), $x \ln R^{C}$ (empty inverted triangles), and the uninoculated control (filled circles). Error bars indicate standard deviations calculated from 20 plants for each treatment. 
indicate that activation of $\mathrm{X} \ln \mathrm{R}$ by inducing carbon sources in $F$. oxysporum occurs at both the transcriptional and post-translational level. Although the $x \ln R$ transcript level increases significantly during induction by OSX (probably via an autoregulatory process, as mentioned above), this higher amount of transcript apparently is not sufficient to activate expression of the target genes. This conclusion is supported by two lines of evidence from the $x \ln R^{C}$ overexpressing strain. First, although the $x \ln R^{C}$ strain has dramatically higher $x \ln R$ transcript levels than the wild-type strain (Fig. 3D), xylanase gene expression and extracellular xylanase activity are not significantly enhanced (Fig. 4). Second, the $x \ln R^{C}$ strain accumulates $x \ln R$ transcript even under noninducing or repressing conditions (e.g., in the presence of glucose), but this does not result in significant amounts of extracellular xylanase activity under these conditions. These results suggest that post-translational modifications are required to obtain active XlnR protein from an inactive precursor, and that it is the amount of active XlnR, not the amount of total protein, that is limiting for activation of xylanase gene expression. In addition, XlnR activity also may be modulated by interaction with other transcriptional regulators such as Ace1, Ace2, or the Hap complex reported in T. reesei (Rauscher et al. 2006). Interestingly, in contrast to the situation in culture, during plant infection, the $x \ln R^{C}$ strain had approximately sixfold higher transcript levels of the $x y l 3$ and $x y l 4$ genes than the wild type. In the saprophyte A. niger, expression of genes controlled by XlnR also was increased in strains harboring multiple copies of the $x \ln R$ gene (Gielkens et al. 1999; van Peij et al. 1998a). It is possible that the activating mechanism of XlnR in F. oxysporum operates more efficiently during plant infection than under conditions of saprophytic growth. More studies are needed to elucidate the molecular bases of this differential behavior in vitro and in planta.

Expression of xylanase genes in $F$. oxysporum appears to be regulated by ambient $\mathrm{pH}$, because transcription of $x y l 4$ is increased at alkaline versus acidic pH (Gómez-Gómez et al. 2002). By contrast, we found that expression of $x \ln R$ is not $\mathrm{pH}$ dependent. Thus, the effect of ambient $\mathrm{pH}$ on expression of structural xylanase genes is exerted directly at the transcriptional level of these genes, rather than indirectly via expression of the XlnR activator. A similar effect of $\mathrm{pH}$ regulation on $\mathrm{xy}-$ lanase gene expression has been reported in Aspergillus spp. (MacCabe et al. 1998).

Real-time RT-PCR analysis showed that the transcriptional activation of xylanase genes and of the $x \ln R$ gene itself by OSX is completely abolished in the presence of glucose. This finding confirms and extends the results from previous studies obtained by Northern analysis (Gómez-Gómez et al. 2001, 2002; RuizRoldán et al. 1999). Glucose repression of xylanase genes in Aspergillus spp. is mediated by the carbon catabolite repressor CreA (de Vries et al. 1999; Orejas et al. 2001) whereas, in $T$. reesei, some but not all of the major hydrolases are under direct Cre1 control (Mach et al. 1996). Currently, there is no formal proof that CreA plays a similar role in Fusarium spp., although the fact that the promoter regions of $x \ln R$ as well as of the $x y l 3$, $x y l 4$, and $x y l 5$ genes contain putative CreA-binding sequences suggests that CreA may mediate glucose repression of the xylanolytic system in F. oxysporum. However, further experimental data are needed to confirm this hypothesis.

The nature of molecules responsible for the inducing effect of OSX is currently unknown. Because xylan polymers are too large to enter the cell, small molecules obtained from degradation of the polymer are likely to function in activation of the genes. In fact, the monomer xylose has been shown to act as an efficient inducer of xylanase gene expression in Aspergillus spp. (de Vries et al. 1999). By contrast, in F. oxysporum, there is conflicting evidence as to the role of xylose. Whereas xylose triggered transcriptional activation of the $x \ln R$ gene (Figs. 1B and 3D), it failed to induce expression of xylanase genes (Fig. 1C) or extracellular xylanase activity (Fig. 4C). This result suggests that other compounds may act as the major inducers of xylanase genes in F. oxysporum. Indeed, thin-layer chromatography analysis of the products from enzymatic hydrolysis of OSX revealed the presence of derivatives other than xylose (Fig. 2C). Whether these compounds act as inducers of xylanases in $F$. oxysporum and how their presence is detected by the fungal cell remains to be determined.

\section{Role of $X \ln R$ in virulence.}

During colonization of the host plant, F. oxysporum expresses multiple genes encoding cell-wall-degrading enzymes that are secreted into the fungus-plant interface (Di Pietro et al. 2003). A major setback in studying the role of these enzymes is their regulatory complexity and functional redundancy. So far, four structural xylanase genes have been cloned from $F$. oxysporum and all of these were expressed during infection of tomato plants (Gómez-Gómez et al. 2001, 2002; Ruiz-Roldán et al. 1999; this work). In the related species F. graminearum, whose genome has been sequenced, expression of no less than 30 different xylanase genes could be detected during growth on different carbon sources (Hatsch et al. 2006). Although individual xylanases are dispensable for virulence, it still appears possible that, collectively, these enzymes could contribute to the infection process (Gómez-Gómez et al. 2001, 2002). In the present study, a mutant lacking XlnR, the major transcriptional activator of xylanase genes, was impaired in activation of the xylanolytic system but still exhibited full virulence on tomato plants. Real-time RT-PCR confirmed that the expression of $x y l 3$ and xyl4 xylanase genes during the infection was basically undetectable in the $\Delta x \ln R$ mutant. We cannot rule out the presence of other xylanase genes whose expression is independent of XlnR. At present, there is little evidence for the existence of such genes, because total xylanase activity in the $\Delta x \ln R$ mutant was extremely low. Nevertheless, the fact that the $\Delta x \ln R$ mutant was able to grow on xylan as the sole carbon source indicates that the residual xylanase activity of the mutant still could be sufficient to break down the plant cell wall. Thus, although the results of this study clearly demonstrate that $X \operatorname{lnR}$, the major transcriptional activator of xylanase genes, is not essential for virulence of $F$. oxysporum, we cannot conclusively rule out a possible role of xylanases in plant infection.

\section{MATERIALS AND METHODS}

Fungal strains and culture conditions.

F. oxysporum f. sp. lycopersici strain 4287 (race 2) was stored as microconidial suspension in $30 \%$ glycerol at $-80^{\circ} \mathrm{C}$. For extraction of DNA, mycelium was obtained from cultures grown in potato dextrose broth (PDB; Difco Laboratories, Detroit) in Erlenmeyer flasks on a rotary shaker at $170 \mathrm{rpm}$ and $28^{\circ} \mathrm{C}$ as described (Di Pietro and Roncero 1998). For analysis of gene expression, freshly obtained microconidia were germinated for $14 \mathrm{~h}$ in PDB, washed twice in sterile water, and transferred to synthetic medium (SM) (Di Pietro and Roncero 1998 ), or to SM buffered at pH 5.5, 6.5, or 8.0 with $\mathrm{NaH}_{2} \mathrm{PO}_{4}$ and $\mathrm{Na}_{2} \mathrm{HPO}_{4}$, supplemented with the appropriate carbon sources. Tomato vascular tissue was collected as described previously (Di Pietro and Roncero 1998). Xylanase and cellulase activities were detected by the production of a clear halo on plates containing $0.15 \%$ azoxylan (Megazyme, Bray, Ireland) or $0.5 \%$ carboxymethyl cellulose (Sigma, St. Louis), respectively. The strains were point inoculated and allowed to grow at $28^{\circ} \mathrm{C}$ for 3 days. 
Nucleic acid manipulations and cloning of the $F$. oxysporum $x \ln R$ gene.

Total RNA and genomic DNA were extracted from $F$. oxysporum mycelium following previously reported protocols (Chomczynski and Sacchi 1987; Raeder and Broda 1985). Southern and Northern blot analyses and probe labeling were carried out as described (Di Pietro and Roncero 1998), using a nonisotopic digoxigenin-labeling kit (Roche Diagnostics SL, Barcelona, Spain). Other routine DNA procedures were performed as described in standard protocols (Sambrook and Russell 2001).

Genomic DNA of $F$. oxysporum strain 4287 was used for PCR amplification on a Perkin-Elmer Gene Amp system 2400 with the degenerated primers xlnR3 (5'-GNGCNTGYGAYCA RTGYAAYCARYT- $3^{\prime}$ ) and xlnR8 (5'-TGYTGYTGYTCNCC RAARTCYTCNGG-3') derived from highly conserved regions of the $x \ln R$ genes from $A$. oryzae, A. niger, and A. kawachii (GenBank accession numbers AB042843, AJ001909, and AB064658, respectively). PCRs were performed routinely with the long-template PCR system (Roche Diagnostics, SL). The amplified 2.5-kb fragment was cloned into the pGEM-T vector (Promega Corp., Madison, WI, U.S.A.) and used as a probe to screen a $\lambda E M B L 3$ genomic library of $F$. oxysporum $\mathrm{f}$. sp. lycopersici isolate 4287. A cDNA clone of $x \ln R$ was isolated by screening a $\lambda$-ZAP cDNA library of $F$. oxysporum $\mathrm{f}$. sp. lycopersici isolate 4287. Sequencing of both DNA strands of the obtained clones was performed at the Servicio de Secuenciación Automática de DNA, SCAI (University of Córdoba, Spain) using the Dyedeoxy Terminator Cycle Sequencing Kit (Applied Biosystems, Foster City, CA, U.S.A.) on an ABI Prism 377 Genetic Analyzer apparatus (Applied Biosystems). DNA and protein sequence databases were searched using the BLAST algorithm (Altschul et al. 1990).

\section{Construction of plasmid vectors and fungal transformation.}

Gene replacement vector $\mathrm{pD} x \ln R$ was constructed as follows. A BamHI site was introduced at position 254 of the $x \ln R$ coding region, using the overlapping method (Sambrook and Russell 2001) with the primers xlnR23B (5'-AGCACTTCAGGATCC ATCAGGAGAA- $3^{\prime}$ ) and $x \ln 24 \mathrm{~B}$ (5'-TCTCCTGATGGATC CTGAAGTGCTG-3'), and the final product was cloned into pGEM-T. A 2.1-kb BamHI-fragment, containing the binuclear zinc cluster, was replaced with a 1.8-kb BamHI fragment containing the hygromycin $\mathrm{B}$ resistance gene under the control of a $C$. heterostrophus promoter (Turgeon et al. 1987). A linear fragment containing the interrupted $x \ln R$ allele was amplified with primers $x \ln R 18$ (5'-CAGCACTCAACTCACATACAA$\left.3^{\prime}\right)$ and $x \operatorname{lnR} 19$ (5'-GTTTACCTACCTCTTACATCTA-3') and used to transform protoplasts of $F$. oxysporum f. sp. lycopersici as described (Di Pietro and Roncero 1998). Hygromycin-resistant transformants routinely were subjected to two consecutive rounds of single sporing and stored as microconidia at $-80^{\circ} \mathrm{C}$. For complementation experiments, a 5.2-kb DNA fragment encompassing the entire $x \ln R$ gene was obtained by PCR with primers xlnR33X (5'-TCTAGACCGTCTCCCTTTCCCCCC-3') and $x \operatorname{lnR} 36 \mathrm{X}$ (5'-CGAACCATCTCTAGAAAGAAACG-3'), using $F$. oxysporum genomic DNA as a template. The obtained fragment was introduced into protoplasts of a $\Delta x \ln R$ mutant by cotransformation with the plasmid pAN8-1 carrying the phleomycin resistance cassette (Mattern et al. 1988) as previously described (Di Pietro et al. 2001). To generate a highly expressed allele of $x \ln R$, the coding region was fused to the strong constitutive A. nidulans gpdA promoter (Punt et al. 1990). To this end, a 3.3-kb fragment containing the complete ORF of $x \ln R$ was amplified by PCR from $F$. oxysporum genomic DNA using primers $x \operatorname{lnR} 29 \mathrm{X}$ (5'-TCTAGAATGCTATCTAATCCAC-3') and $x \operatorname{lnR} 18$ (5'-CAGCACTCAACTCACATACAA-3'), cloned into pGEM-T and a XbaI-NotI fragment was isolated and cloned into pGEM-T containing $1.4 \mathrm{~kb}$ of the $g p d A$ promoter. The complete promoter fusion construct was excised with HindIII-NotI and cloned into the pBKS vector containing the hygromycin B resistance cassette, and protoplasts of $F$. oxysporum were transformed with the construct as described above.

\section{Determination of xylanase activity.}

For determination of total xylanase activity, fungal strains were grown for $24 \mathrm{~h}$ in liquid SM with $1 \%$ OSX as the sole carbon source. Supernatants were harvested by Millipore filtration, dialyzed against water, and assayed for xylanase activity as described (Ruiz Roldan et al. 1997). Specific activity was expressed in nkatal (defined as the amount of enzyme that releases $1 \mathrm{nmol}$ of reducing sugar per second) per milligram of protein.

\section{Real-time RT-PCR.}

Total RNA $(1 \mu \mathrm{g})$ was reverse transcribed using SuperScript II (Invitrogen, Carlsbad, CA, U.S.A.) as indicated by the manufacturer. Real-time PCR reactions of the cDNA were performed with $25-\mu \mathrm{l}$ reaction volumes with the SYBR Green I dye (Molecular Probes, Sunnyvale, CA, U.S.A.), $1 \mu \mathrm{l}$ of cDNA template, $7.5 \mathrm{pmol}$ of each primer, $160 \mathrm{mM}$ dNTPs, $2 \mathrm{mM} \mathrm{MgCl}_{2}, 0.5 \mathrm{U}$ of BioTaq, using an iQ Cycler Real-Time PCR detection system (BioRad Laboratories, Hercules, CA, U.S.A.). PCRs for each gene were done in triplicate and data were analyzed with the gene expression software supplied with the iQ Cycler system. Real-time PCR primers for $x y l 3, x y l 4, x y l 5, x \ln R$, and act 1 genes are indicated in Supplemental Table 1. Melting curve analysis was performed after the PCR reaction was complete to confirm the absence of nonspecific amplification products. The average cycle threshold of actin under each condition was used to normalize the average cycle threshold for each gene. The relative expression ratio of each target gene was calculated as described (Pfaffl 2001).

\section{Thin-layer chromatography of the hydrolysis products.}

The products of enzymatic hydrolysis from fungal cultures grown on SM with $1 \%$ OSX were examined by thin-layer chromatography on silica gel precoated plates (Sigma). Culture supernatant $(500 \mu \mathrm{l})$ was removed at the indicated time points, lyophilized, and resuspended in $50 \mu \mathrm{l}$ of water. Aliquots of 10 $\mu \mathrm{l}$ were subjected to thin-layer chromatography using ethyl acetate/acetic acetic/water (18:7:8, vol/vol/vol) as solvents, and sprayed with $2.5 \%$ aniline phenylamine in acetone to detect hydrolysis products. D-xylose was run as a standard.

\section{Plant infection assays.}

Tomato infection assays were performed as described (Di Pietro and Roncero 1998). Briefly, 2-week-old tomato seedlings (cv. Vemar) were inoculated with $F$. oxysporum strains by immersing the roots into a microconidial suspension, planted in vermiculite, and maintained in a growth chamber. At different times after inoculation, severity of disease symptoms was recorded using an index ranging from 1 (healthy plant) to 5 (dead plant). Twenty plants were used for each treatment. RNA was extracted from roots and stems of inoculated and control plants and fungal gene expression was determined by RT-PCR analysis as described (Di Pietro and Roncero 1998). Experiments were performed at least twice with similar results.

\section{ACKNOWLEDGMENTS}

This work was supported by grant BIO2004-00276 from the Spanish Ministerio de Ciencia y Tecnología. F. Calero-Nieto was supported by a Ph.D. fellowship from the Spanish Ministerio de Educación, Cultura y De- 
porte. A. Di Pietro was the recipient of a Ramón y Cajal grant from the Ministerio de Ciencia y Tecnología. The authors are grateful to Isabel Caballero, Universidad de Córdoba, for valuable technical assistance.

\section{LITERATURE CITED}

Altschul, S. F. Gish, W., Miller, W., Myers, C. W., and Lipman, D. J. 1990. Basic local alignment search tool. J. Mol. Biol. 215:403-410.

Apel, P. C., Panaccione, D. G., Holden, F. R., and Walton, J. D. 1993. Cloning and targeted gene disruption of $X Y L 1$, a $\beta$ 1,4-xylanase gene from the maize pathogen Cochliobolus carbonum. Mol. Plant-Microbe Interact. 6:467-473

Apel-Birkhold, P., and Walton, J. 1996. Cloning, disruption, and expression of two endo-beta 1, 4-xylanase genes, XYL2 and XYL3, from Cochliobolus carbonum. Appl. Environ. Microbiol. 62:4129-4135.

Belien, T., Van Campenhout, S., Robben, J., and Volckaert, G. 2006. Microbial endoxylanases: Effective weapons to breach the plant cell wall barrier or, rather, triggers of plant defense systems? Mol. Plant-Microbe Interact. 19:1072-1081.

Brakhage, A. A., Andrianopoulos, A., Kato, M., Steidl, S., Davis, M. A., Tsukagoshi, N., and Hynes, M. J. 1999. HAP-Like CCAAT-binding complexes in filamentous fungi: Implications for biotechnology. Fungal Genet. Biol. 27:243-252.

Brito, N., Espino, J. J., and Gonzalez, C. 2006. The endo- $\beta-1,4-x y l a n a s e$ Xyn11A is required for virulence in Botrytis cinerea. Mol. PlantMicrobe Interact. 19:25-32.

Chomczynski, P., and Sacchi, N. 1987. Single-step method of RNA isolation by acid guanidinium thiocyanate-phenol-chloroform extraction. Anal. Biochem. 162:156-159.

de Vries, R. P., and Visser, J. 1999. Regulation of the feruloyl esterase (faeA) gene from Aspergillus niger. Appl. Environ. Microbiol. 65:55005503.

de Vries, R. P., and Visser, J. 2001. Aspergillus enzymes involved in degradation of plant cell wall polysaccharides. Microbiol. Mol. Biol. Rev. 65:497-522.

de Vries, R. P., Visser, J., and de Graaff, L. H. 1999. CreA modulates the XlnR-induced expression on xylose of Aspergillus niger genes involved in xylan degradation. Res. Microbiol. 150:281-285.

Di Pietro, A., and Roncero, M. I. G. 1998. Cloning, expression, and role in pathogenicity of $p g 1$ encoding the major extracellular endopolygalacturonase of the vascular wilt pathogen Fusarium oxysporum. Mol. PlantMicrobe Interact. 11:91-98.

Di Pietro, A., García-Maceira, F. I., Meglecz, E., and Roncero, M. I. G. 2001. A MAP kinase of the vascular wilt fungus Fusarium oxysporum is essential for root penetration and pathogenesis. Mol. Microbiol. 39:1140-1152

Di Pietro, A., Madrid, M. P., Caracuel, Z., Delgado-Jarana, J., and Roncero, M. I. G. 2003. Fusarium oxysporum: Exploring the molecular arsenal of a vascular wilt fungus. Mol. Plant Pathol. 4:315-325.

Gielkens, M. M., Dekkers, E., Visser, J., and de Graaff, L. H. 1999. Two cellobiohydrolase-encoding genes from Aspergillus niger require D-xylose and the xylanolytic transcriptional activator XlnR for their expression. Appl. Environ. Microbiol. 65:4340-4345.

Gómez-Gómez, E., Roncero, M. I. G., Di Pietro, A., and Hera, C. 2001 Molecular characterization of a novel endo-beta-1,4-xylanase gene from the vascular wilt fungus Fusarium oxysporum. Curr. Genet. 40:268-275

Gómez-Gómez, E., Ruiz-Roldán, M. C., Di Pietro, A., Roncero, M. I. G., and Hera, C. 2002. Role in pathogenesis of two endo-beta-1,4-xylanase genes from the vascular wilt fungus Fusarium oxysporum. Fungal Genet. Biol. 35:213-222.

Hasper, A. A., Visser, J., and de Graaff, L. H. 2000. The Aspergillus niger transcriptional activator $\mathrm{X} \operatorname{lnR}$, which is involved in the degradation of the polysaccharides xylan and cellulose, also regulates D-xylose reductase gene expression. Mol. Microbiol. 36:193-200.

Hatsch, D., Phalip, V., Petkovski, E., and Jeltsch, J. M. 2006. Fusarium graminearum on plant cell wall: No fewer than 30 xylanase genes transcribed. Biochem. Biophys. Res. Commun. 345:959-966.

MacCabe, A. P., Orejas, M., Perez-Gonzalez, J. A., and Ramon, D. 1998. Opposite patterns of expression of two Aspergillus nidulans xylanase genes with respect to ambient pH. J. Bacteriol. 180:1331-1333.

Mach, R. L., Strauss, J., Zeilinger, S., Schindler, M., and Kubicek, C. P. 1996. Carbon catabolite repression of xylanase I (xyn1) gene expression in Trichoderma reesei. Mol. Microbiol. 21:1273-1281.

Marmorstein, R., and Harrison, S. C. 1994. Crystal structure of a PPR1DNA complex: DNA recognition by proteins containing a Zn2Cys6 binuclear cluster. Genes Dev. 8:2504-2512.

Marui, J., Tanaka, A., Mimura, S., de Graaff, L. H., Visser, J., Kitamoto,
N., Kato, M., Kobayashi, T., and Tsukagoshi, N. 2002. A transcriptional activator, AoXlnR, controls the expression of genes encoding xylanolytic enzymes in Aspergillus oryzae. Fungal Genet. Biol. 35:157-169.

Mattern, I. E., Punt, P. J., and van den Hondel, C. A. 1988. A vector for Aspergillus transformation conferring phleomycin resistance. Fungal Genet. Newsl. 35:25.

Nikolaev, I., Cochet, M. F., Lenouvel, F., and Felenbok, B. 1999. A single amino acid, outside the AlcR zinc binuclear cluster, is involved in DNA binding and in transcriptional regulation of the alc genes in Aspergillus nidulans. Mol. Microbiol. 31:1115-1124.

Orejas, M., MacCabe, A. P., Pérez-González, J. A., Kumar, S., and Ramón, D. 2001. The wide-domain carbon catabolite repressor CreA indirectly controls expression of the Aspergillus nidulans $x \ln B$ gene, encoding the acidic endo-beta-(1,4)-xylanase $\mathrm{X}_{(24)}$. J. Bacteriol. 183:1517-1523.

Pfaffl, M. W. 2001. A new mathematical model for relative quantification in real-time RT-PCR. Nucleic Acids Res. 29:e45.

Punt, P. J., Dingemanse, M. A., Kuyvenhoven, A., Soede, R. D., Pouwels, P. H., and van den Hondel, C. A. 1990. Functional elements in the promoter region of the Aspergillus nidulans gpdA gene encoding glyceraldehyde-3-phosphate dehydrogenase. Gene 93:101-109.

Raeder, U., and Broda, P. 1985. Rapid preparation of DNA from filamentous fungi. Lett. Appl. Microbiol. 1:17-20.

Rauscher, R., Wurleitner, E., Wacenovsky, C., Aro, N., Stricker, A. R., Zeilinger, S., Kubicek, C. P., Penttila, M., and Mach, R. L. 2006. Transcriptional regulation of xyn 1, encoding xylanase I, in Hypocrea jecorina. Eukaryot. Cell 5:447-456.

Ruiz-Roldán, M. C., Di Pietro, A., and Roncero, M. I. G. 1997. Purification and characterization of an acidic endo b-1,4-xylanase from the tomato vascular pathogen Fusarium oxysporum f. sp. lycopersici. FEMS (Fed. Eur. Microbiol. Soc.) Microbiol. Lett. 148:75-92.

Ruiz-Roldán, M. C., Di Pietro, A., Huertas-González, M. D., and Roncero, M. I. G. 1999. Two xylanase genes of the vascular wilt pathogen Fusarium oxysporum are differentially expressed during infection of tomato plants. Mol. Gen. Genet. 261:530-536.

Sambrook, J., and Russell D. W. 2001. Molecular Cloning: A Laboratory Manual. Cold Spring Harbor Laboratory Press, Cold Spring Harbor, NY, U.S.A.

Schjerling, P., and Holmberg, S. 1996. Comparative amino acid sequence analysis of the C6 zinc cluster family of transcriptional regulators. Nucleic Acids Res. 24:4599-4607.

Stricker, A. R., Grosstessner-Hain, K., Würleitner, E., and Mach, R. L. 2006. Xyr 1 (xylanase regulator 1) regulates both the hydrolytic enzyme system and the xylose metabolism in Hypocrea jecorina. Eukaryot. Cell 5:2128-2137

Suárez, T., de Queiroz, M. V., Oestreicher, N., and Scazzocchio, C. 1995. The sequence and binding specificity of UaY, the specific regulator of the purine utilization pathway in Aspergillus nidulans, suggest an evolutionary relationship with the PPR1 protein of Saccharomyces cerevisiae. EMBO (Eur. Mol. Biol. Organ.) J. 14:1453-1467.

Tonukari, N. J., Scott-Craig, J. S., and Walton, J. D. 2000. The Cochliobolus carbonum SNF1 gene is required for cell wall-degrading enzyme expression and virulence on maize. Plant Cell 12:237-247.

Turgeon, B. G., Garber, R. C., and Yoder, O. C. 1987. Development of a fungal transformation system based on selection of sequences with promoter activity. Mol. Cell. Biol. 7:3297-3305.

van Peij, N. N., Gielkens, M. M., de Vries, R. P., Visser, J. and de Graaff, L. H. 1998a. The transcriptional activator XlnR regulates both xylanolytic and endoglucanase gene expression in Aspergillus niger. Appl. Environ. Microbiol. 64:3615-3619.

van Peij, N. N., Visser, J., and de Graaff, L. H. 1998b. Isolation and analysis of $x \ln \mathrm{R}$, encoding a transcriptional activator co-ordinating $x y-$ lanolytic expression in Aspergillus niger. Mol. Microbiol. 27:131-142.

Walton, J. D. 1994. Deconstructing the cell wall. Plant Physiol. 104:11131118.

Wong, K. K., Tan, L. U., and Saddler, J. N. 1988. Multiplicity of beta-1,4xylanase in microorganisms: Functions and applications. Microbiol. Rev. 52:305-317.

Wu, S. C., Halley, J. E., Luttig, C., Fernekes, L. M., Gutierrez-Sanchez, G., Darvill, A. G., and Albersheim, P. 2006. Identification of an endobeta-1,4-D-xylanase from Magnaporthe grisea by gene knockout analysis, purification, and heterologous expression. Appl. Environ. Microbiol. 72:986-993.

\section{AUTHOR-RECOMMENDED INTERNET RESOURCES}

Broad Institute of MIT and Harvard Fusarium graminearum sequencing project: www.broad.mit.edu

Softberry, Inc. website: www.softberry.com 